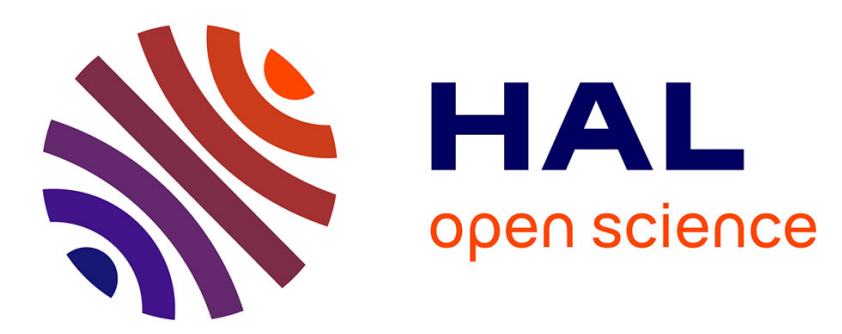

\title{
Handover Enhancement for LTE-Advanced and Beyond Heterogeneous Cellular Networks
}

\author{
Baha Uddin Kazi, Gabriel Wainer
}

\section{To cite this version:}

Baha Uddin Kazi, Gabriel Wainer. Handover Enhancement for LTE-Advanced and Beyond Heterogeneous Cellular Networks. 2017 International Symposium on Performance Evaluation of Computer and Telecommunication Systems (SPECTS), Jul 2017, Seattle, United States. hal-01949220

\section{HAL Id: hal-01949220 \\ https://hal.science/hal-01949220}

Submitted on 9 Dec 2018

HAL is a multi-disciplinary open access archive for the deposit and dissemination of scientific research documents, whether they are published or not. The documents may come from teaching and research institutions in France or abroad, or from public or private research centers.
L'archive ouverte pluridisciplinaire HAL, est destinée au dépôt et à la diffusion de documents scientifiques de niveau recherche, publiés ou non, émanant des établissements d'enseignement et de recherche français ou étrangers, des laboratoires publics ou privés. 


\title{
Handover Enhancement for LTE-Advanced and Beyond Heterogeneous Cellular Networks
}

\author{
Baha Uddin Kazi and Gabriel Wainer \\ Dept. of Systems and Computer Engineering \\ Carleton University, Ottawa, ON, Canada \\ \{bahauddinkazi,gwainer\}@sce.carleton.ca
}

\begin{abstract}
Heterogeneous networks (HetNets) are considered a promising cellular network architecture to provide services to the massive number of subscribers. However, in heterogeneous networks, as cell size becomes smaller, the number of handovers and handover failure increase significantly. Therefore, mobility management becomes an important issue in HetNets. In this research, we analyzed the handover parameters, and proposed a novel handover method for heterogeneous cellular networks to minimize the number of handovers and handover failure. In the proposed method, we considered dual connectivity with control and data plane split and Coordinated Multipoint (CoMP) transmission to optimize the handover parameters. The reduction of handover improves the network performance and the handover failure reduction improves the user experience. The simulation results show that the proposed handover process significantly reduces the number of handovers in heterogeneous cellular networks.
\end{abstract}

Keywords-Handover, Heterogeneous networks, CoMP, Duel connectivity, LTE-Advanced, DEVS.

\section{INTRODUCTION}

In recent years, the demand of data traffic and the number of subscribers in cellular networks is increasing rapidly. The number of mobile broadband subscriptions is growing globally by around $25 \%$ each year, and it is predicted to reach 7.7 billion by 2021 [1]. More than 50 billion wireless devices are predicted to be connected to the cellular networks by 2020 and network's data traffic is expected to reach 351 Exabyte by 2025 $[2,3,4]$. Moreover, $5 \mathrm{G}$ networks are expected to provide approximately a system capacity 1000 times higher, 10 times the data rates, 25 times the average cell throughput and 5 times reduced latency when compared to the $4 \mathrm{G}$ networks $[5,3,6]$. Therefore, to achieve the goals of next generation cellular networks it is essential to improve the capacity of mobile networks by overcoming the existing challenges.

In this context, network densification or heterogeneous networks (HetNets) are considered as an effective method to improve the capacity of cellular networks [7, 8, 6]. HetNets consist of coexisting macro-cells and low-power nodes for small cells such as Pico-cells and Femtocells. As the size of cells decreases, the number of cells will increase, providing service to more users. However, there are two issues arising when the cell size is reduced: mobility and interference [9]. Therefore, users in the cell edge experience frequent handover (HO), and thus the handover failure (HOF) rate also increase.
The 3rd Generation Partnership Project (3GPP), telecommunications standardization body showed that the increase in the number of handover in small cell network compared to macro only networks can be $120 \%-140 \%$, depending on the speed of the user equipment (UE) [7]. Consequently, to realize the potential coverage and capacity benefits with small cells, adequate mobility management is needed, and this has become a major technical challenge in HetNets.

The handover process is used to support the seamless mobility of the UEs. The HO process makes UEs in active mode to be transferred from the serving cell to the neighboring cell with the strongest received power, and the user is not aware, as shown in figure 1 . In conventional homogeneous cellular networks, typically same set of handover parameters are used in all over the networks. However, in HetNets, if the same set of parameters is used for all UEs and all the types of cells, there is a possibility to degrade the mobility performance [10]. The increase in the number of handovers will increase the control overhead and the switching load into the network that will eventually decrease the network performance. Maintaining low $\mathrm{HOF}$ rate is also important for better user experience. Therefore, it is essential to analyze the handover parameters and to enhance them for heterogeneous cellular networks.

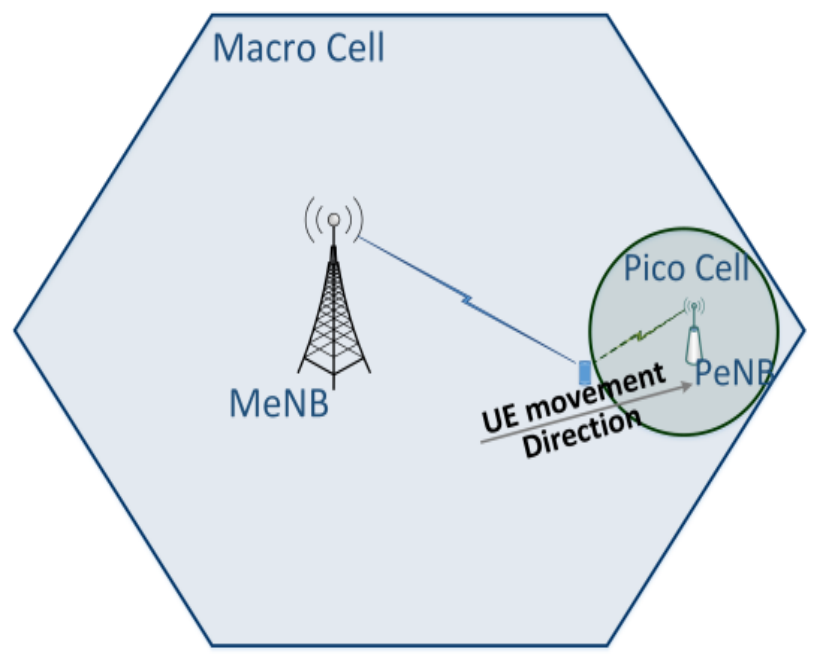

Fig. 1: High-level handover architecture

In this research, we proposed a novel handover method named EHoLM: Enhanced Handover for Low and Moderate speed UEs of LTE-Advanced and beyond heterogeneous cellu- 
lar networks. In the EHoLM, we use control plane and data plane separation for the UEs, who are within the CoMP transmission and reception. The CoMP transmission reduce the inter-cell interference, hence signal quality of serving cell remains better than conventional transmission for the UE. Therefore, in the EHoLM handover, handover criteria will not satisfy until a UE moves from CoMP to no CoMP region of different eNB instead of the conventional handover criteria (A3 event). Simulation results also clearly shows that the EHoLM handover method reduces the number of handovers. The reduction of handover will improve the network performance as well as the reduction of handover failure rate will improve the user experience.

The rest of the paper is organized as follows. We discuss background and related works in section 2 . In section 3 , we briefly discuss about the handover procedure in the standardization process of LTE and LTE-A mobile networks. The EHoLM handover process is presented in section 4. In section 5 , we present the simulation model of the EHoLM handover procedure. We use the discrete event system (DEVS) formalism to model the EHoLM. Simulation scenarios and assumptions are presented in section 6 . In section 7 , the simulation results are presented. Finally, we conclude with future works in the last section.

\section{BACKGROUND AND RELATED WORKS}

To improve the capacity of cellular networks, 3GPP considered a number of technologies including multiple inputs multiple outputs (MIMO), mm-wave communication and heterogeneous networks (HetNets) $[2,8,11]$ in LTE-Advanced and beyond. Among the various techniques, heterogeneous networks have been adapted as key technology to provide services a massive number of users $[5,12,13,8,14]$. HetNets are comprised of different types of small cells with different capabilities. These include Remote Radio Head (RRH), Pico eNB (PeNB) and Home eNB (HeNB). These low power small cells can reduce the load of the macro cells and increase user coverage. However, deployment of these small cells can result in increased interference and mobility [9]. Coordinated multipoint (CoMP) and dual connectivity are two promising technologies to overcome these challenges $[9,13,14,15]$.

Coordinated Multipoint (CoMP) transmission and reception is considered as an effective method to improve the user throughput, especially for cell edge users, by mitigating intercell interference (ICI) $[16,17,12]$. In CoMP enabled systems, the eNBs are grouped into cooperating clusters. The eNBs of each of these clusters exchange information with one another and jointly process signals. Furthermore, multiple User Equipments (UEs) can receive their signals simultaneously from one or more transmission points in a coordinated or jointprocessing manner $[12,18]$. In [19] authors study the performance analysis of the CoMP joint processing (JP) transmission in HetNets scenarios. Geirhofer and Gaal in [20] discuss CoMP in different HetNets scenarios. They also analyze CoMP schemes and the deployment architectures as well as the benefits and drawbacks of them. In [17, 21], we present dynamic coordinator based CoMP control architecture for reducing signaling overhead and feedback latency.
Dual connectivity as we stated earlier, is another promising technology to increase the user throughput as well as to achieve the mobility enhancement $[9,7,15]$. In dual connectivity, UEs can connect two or more eNBs simultaneously in control plane and data plane. 3GPP in [7] suggested three deployment scenarios for heterogeneous networks for further studies. Scenario 1: macro and small cells on the same carrier frequency and connected via non-ideal backhaul. Scenario 2: macro and small cells on different carrier frequency and connected via non-ideal backhaul. Scenario 3: all are small cells on one or more carrier frequencies and connected via non-ideal backhaul. In $[9,15]$, the authors only considered the scenario 2 suggested by 3 GPP in [7]. In our approach, we considered both scenario 1 and 2 suggested by 3 GPP in [7]. In HetNets, we can categories the $\mathrm{HO}$ in four groups according to the types of cells: Macro to Macro handover (MMH), Macro to Pico handover (MPH), Pico to Macro handover (PMH) and Pico to Pico handover (PPH).

In [10], authors presented a review of the handover process, and they identified technical challenges for mobility management in HetNets. 3GPP in [7, 22] discussed about the different deployment scenarios and challenges of small cell enhancements (HetNets). In [11, 23], 3GPP discussed details of the handover process in mobile networks. In $[9,15]$, the authors showed how dual connective could be a promising technology to achieve mobility enhancement.

In order to study this problem, we built a number of models, and simulated them using the $\mathrm{CD}++$ toolkit, which implements DEVS and Cell-DEVS theory [24, 25, 26]. We used this method because DEVS has proved to be a strong mechanism for formal modeling and simulation $(\mathrm{M} \& \mathrm{~S})$ of discrete event dynamic systems. DEVS models are hierarchical and modular, which allows the description of the multiple levels in our approach with ease, and enhances the reusability of a model. It reduces the computational time by reducing the number of calculations for a given accuracy. The same model could be extended with different DEVS based simulators, allowing for portability and interoperability at a high level of abstraction. Finally, the use of formal modeling techniques enables automated model verification [27]. Considering the advantages, we used DEVS to build a number of models to study the performance of the EHoLM handover process in heterogeneous cellular networks.

\section{HANDOVER PROCESS IN LTE AND LTE-A}

3GPP specifies a handover procedure and mechanism for LTE and LTE-Advanced mobile networks that supports user's mobility. In LTE-advanced cellular networks, UE-assisted network-controlled handovers are performed [11]. In UE-assisted network-controlled handovers, the serving eNB makes the decision to move from one cell to another based on the measurement report $(\mathrm{MR})$ received from the UE. The handover procedure of 3GPP LTE and LTE-A is defined in $[11,28]$.

A HO process, in general completes in five steps. 1: the UE measures the downlink signal strength periodically. 2: it processes the measurement. 3: it sends a measurement report (MR) to the serving eNB based on predefined HO criteria. 4: the serving eNB takes the handover decision based on the received MR. 5: the UE receives the handover command from the serving eNB and completes the handover process. 
For modeling, the HO processing of an UE is also divided into 3 states [28]:

- State 1: Before the handover criteria (A3 event) is satisfied.

- State 2: After the handover criteria is satisfied but before the handover command is successfully received by the UE.

- State 3: After the HO command is received by the UE, but before the $\mathrm{HO}$ process is completed successfully.

Figure 2 shows the details states of the handover process.

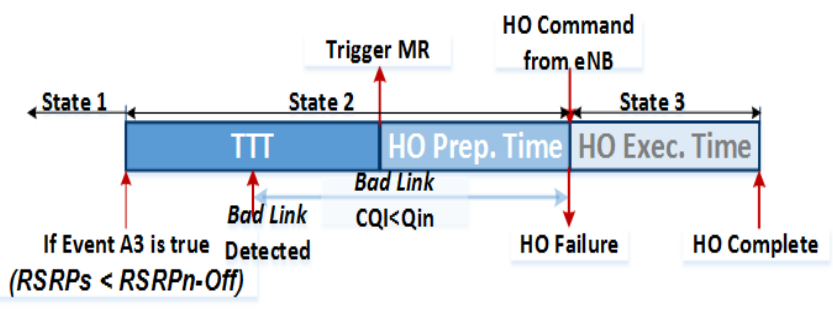

Fig. 2: Block diagram of handover states

The UE calculates reference signal received power (RSRP) every $40 \mathrm{~ms}$ and performs linear average over 5 successive RSRP samples based on the following formula $[29,30,10]$.

$$
M(n)=\frac{1}{5} \sum_{k=0}^{4} R S R P_{l 1}(5 n-k)
$$

Where $R S R P_{l 1}$ is the RSRP sample measured every $40 \mathrm{~ms}$ as mentioned before, $\mathrm{n}$ is the discrete time index of the RSRP sample and $\mathrm{k}$ is the delay index of the filter. As a result, the handover measurement period for an UE in L3 is $200 \mathrm{~ms}$. Once the L3 filtered RSRP of the target cell is higher than the RSRP of serving cell plus A3 offset or hysteresis margin, the UE starts TTT, the Time to Trigger Timer [10, 31].

$$
\text { Event A3: RSRP }{ }_{s}+\text { Off }<\operatorname{RSRP}_{n}
$$

The handover process is performed mainly via the radio resource control (RRC) layer between UE and eNB in the control-plane. The simplified message sequence diagram of LTE and LTE-Advanced handover process is shown in figure 3 [32]. If the $\mathrm{A} 3$ event condition as shown in equation 2 is true throughout the TTT, the UE sends measurement report (MR) to the serving eNB once TTT expires. This MR kicks off the handover preparation phase. The serving eNB issues a handover request message to the target cell. This handover request carries out admission control procedure for the UE in target cell. After completing the admission control, target eNB sends a handover request Ack message to the serving eNB. When the serving eNB receives the handover request Ack, data forwarding from serving eNB to target eNB starts and the serving eNB sends a handover command (RRC Conn. Reconf) to the UE. UE then synchronizes with the target eNB and sends a handover complete message to the target eNB. As a result, intra eNB handover process of the UE is complete, and the target eNB becomes its serving eNB and starts transmitting data to the UE. The new serving eNB sends a path switch request to the serving gateway to inform the core network that it is the new serving eNB for the UE. The serving gateway or the network sends a modify bearer response message to the new serving eNB and switched the downlink data path from previous serving eNB to new serving eNB. Finally, new serving eNB sends message to the old serving eNB requesting to release the resource for the UE.

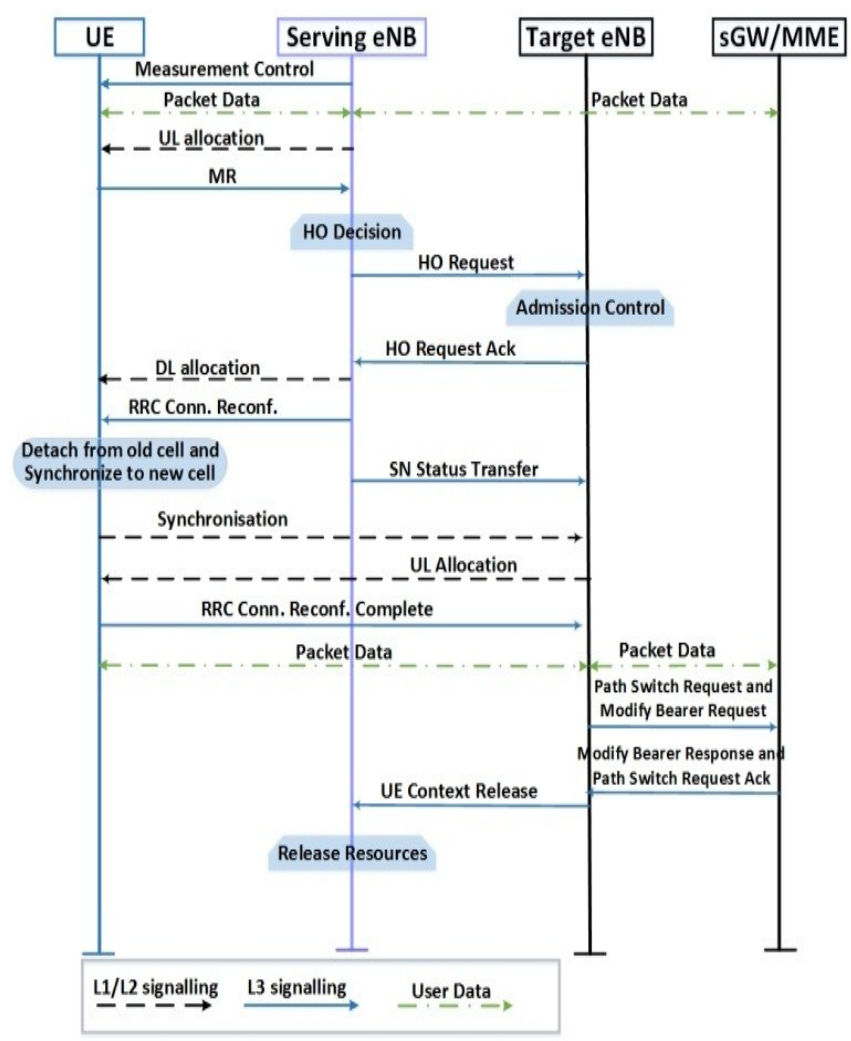

Fig. 3. Message sequence of handover process.

\section{ENHANCED HANDOVER SCHEME FOR HETNETS}

Despite the promising features of HetNets, they have introduced new challenge on mobility management and interference coordination as we mentioned earlier. The handover performance largely depends on the handover parameters such as Time to Trigger (TTT) and A3 offset $[30,10]$. On the other hand, CoMP improves the performance of cell edge users by reducing the interference and serving the UE jointly $[12,32]$. As a result, in CoMP, UEs receives better signal quality than in a conventional transmission. The performance of CoMP also depends on the CoMP threshold. The handover and CoMP both happen on the UEs in the cell edge region and both have their own parameters. Therefore, to achieve the better system performance we need to optimize the handover parameters when the UE served in CoMP cooperation.

Now, consider an UE in the cell edge moving gradually from its serving eNB to a target $\mathrm{eNB}$, and consider that CoMP has been established by more than one eNBs (including the serving and target eNBs) to serve the UE. If the A3 offset $(3 \mathrm{~dB})$ [28] in the handover is less than the CoMP threshold $(6 \mathrm{~dB})[33,21]$, there are some handovers that happened, although the UE is still in CoMP transmission. That is, the UE is handed over to another eNB, but it is still served by all the eNBs together. This is an avoidable handover, which degrades 
the performance of the networks. We want to take the advantage of CoMP, which provides a better signal strength to the cell edge UEs by reducing the ICI as well as dual connectivity that provides control plane and data plane separation for UEs. In EHoLM approach, the handover criteria will not be satisfied until a UE moves from a CoMP to no CoMP region of a different $\mathrm{eNB}$, instead of the conventional handover criteria discussed in equation 2. That is, if an UE moves from a macro cell to a CoMP region, it will stay connected to the macro eNB (serving eNB) until it leaves CoMP and moves to a no CoMP region of another eNB. The EHoLM scheme is shown in figure 4.

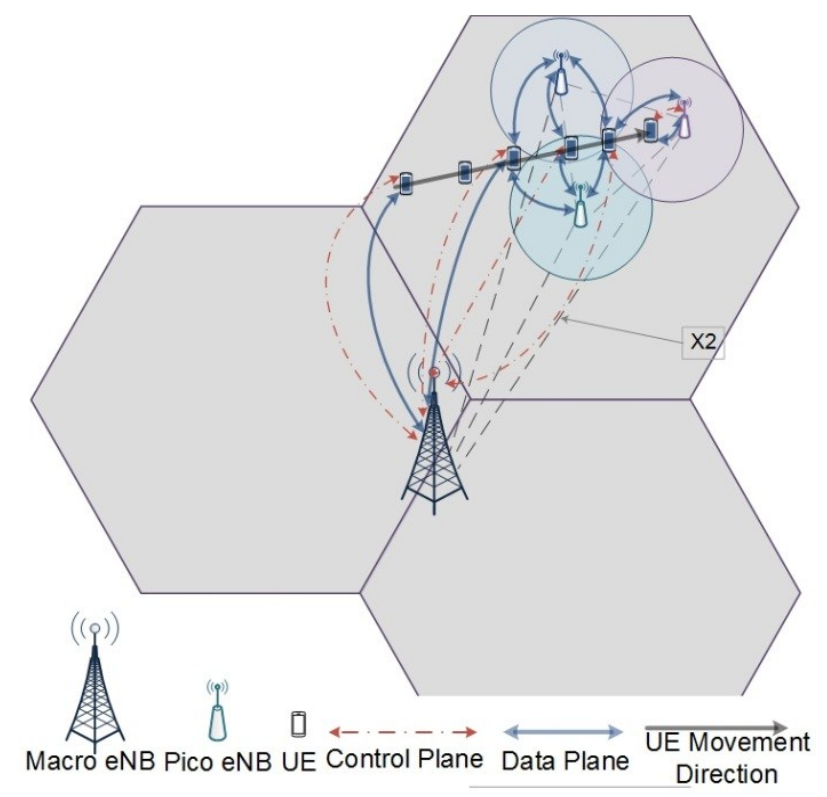

Fig. 4. EHoLM Handover Scheme

In this figure, dashed lines represent the control pane connectivity and solid lines represent the data plane connectivity. Initially, when the UE is in the no CoMP region of the macro eNB, it is connected to the macro eNB control and data planes. Gradually, when the UE moves to the CoMP region, it is served by more than one eNBs in data plane, but it remains connected to the serving eNB in the control plane. Finally, when it moves from the CoMP to the no CoMP region of the pico eNB, it is handed over to the pico eNB.

\section{Modeling EHoLM}

To study the handover procedure with decoupling the control plane and data plane and CoMP, we consider heterogeneous networks as suggested by 3 GPP in $[34,28]$. We designed a DEVS model to examine the performance of EHoLM in LTEAdvanced and beyond heterogeneous mobile networks. A simplified diagram of the structure of the model is shown in figure 5.

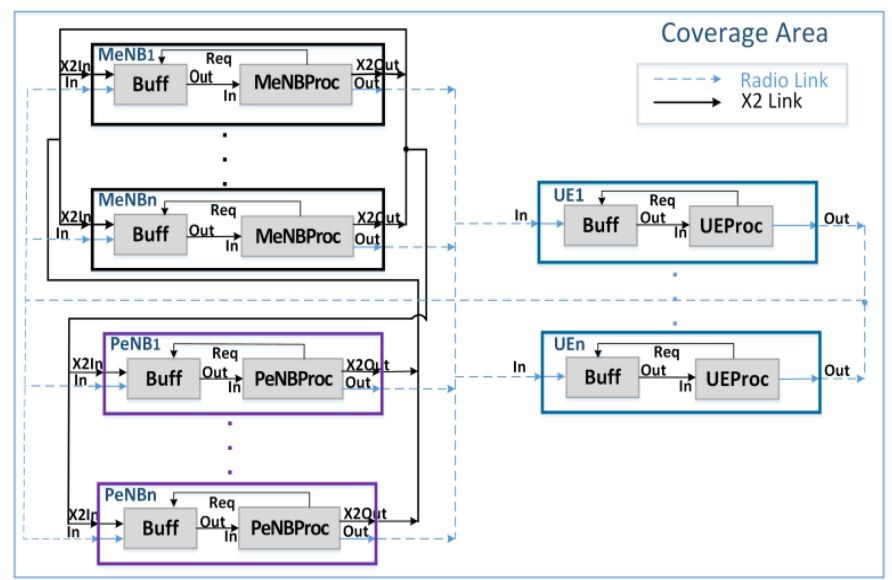

Fig. 5. Simplified DEVS model for CoMP control architecture

In figure 5 above, the black solid links connecting the MeNBs and PeNBs represent the X2 links. The blue dotted line shows the radio link between the MeNBs and PeNBs to UEs. The number of MeNBs, PeNBs and UEs could be changed according to the simulation scenario. The top-level coupled model is the geographic area, which includes a number of cells. Each cell contains one MeNB, multiple PeNBs and many UEs. The numbers of PeNBs and UEs vary based on different scenarios. Each MeNB, PeNB and UE coupled model is composed of two atomic models named Buff and Proc. The UEProc calculates the RSRP based on the formula we discussed above. According to the handover criteria, UEProc generates the MR and sends it to the MeNB Buff or PeNB Buff through the output port (Out). The MeNB Buff acts as a buffer for the MeNB couple model. Once the MeNB receives a message, the MeNB Buff pushes it in a queue. The message is popped out from the queue and forwarded to the MeNBProc when a request is received from the processor. The MeNBProc takes the $\mathrm{HO}$ decision based on the MR it received from the UE and sends the HO request to the target eNB through the output port (X2Out).

\section{SIMULATION SCENARIOS AND ASSUMPTIONS}

To study the potential of the EHoLM handover procedure, we considered the HetNet scenarios suggested in [34, 28]. Figure 6 shows the simplified network architectures of the simulation scenarios we used. The network in figure 6(a) has 1 macro cell and 24 Pico cells. Figure 6(b) shows a HetNet with 19 macro cells and 72 pico cells as suggested by 3GPP [28]. The number of UEs varies, and they are distributed uniformly all over the simulation area. The UEs are considered to be initially connected to the eNBs with strongest received power and move at random directions over the simulation area.

In our simulation scenarios, cells are considered macro and pico cells in an urban area. The propagation model is considered, based on 3GPP standard in $[34,35]$ as follows:

Macro Cell: $128.1+37.6 \log _{10}(\mathrm{~d})$

Pico Cell: $147+36.7 \log _{10}(\mathrm{~d})$

Where $\mathrm{d}$ is the separation between UE and eNB. 

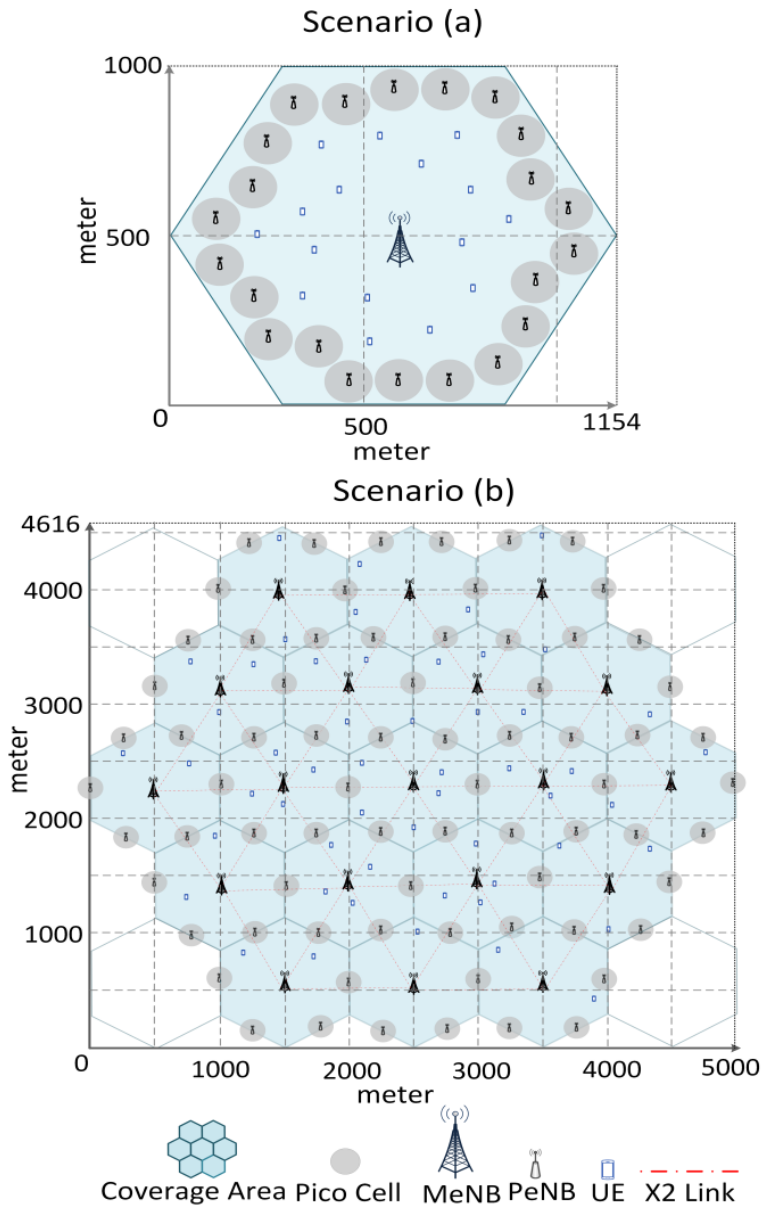

Fig. 6. Simplified Simulation Scenario

We ran a series of simulations on both EHoLM and the conventional handover model, based on the initial conditions summarized in table 1 . We have chosen our simulation parameters based on the 3GPP specifications and different literatures $[34,7,21,33,36,37]$.

TABLE 1: SIMULATION ASSUMPTIONS

\begin{tabular}{|c|c|}
\hline Parameters & Values \\
\hline Number of macro eNBs & 1 and 19 \\
\hline Number of Pico eNBs & 24 and 72 \\
\hline Number of UEs & $25,50,100,200$ \\
\hline UE Distribution & $\begin{array}{l}\text { Uniform: randomly into the simula- } \\
\text { tion area }\end{array}$ \\
\hline Frequency & $2000 \mathrm{MHz}, 35000 \mathrm{MHz}$ \\
\hline Macro eNB Transmit power & $43 \mathrm{dBm}$ \\
\hline Small eNB Transmit Power & $30 \mathrm{dBm}$ \\
\hline Macro Cell Radius & $500 \mathrm{~m}$ \\
\hline Antenna gain & $\begin{array}{c}12 \mathrm{dBi} \text { (Macro eNB), } 05 \mathrm{dBi} \text { (Pico } \\
\text { eNB) and } 0 \mathrm{dBi} \text { (UEs) }\end{array}$ \\
\hline RSRP Sample & Every $40 \mathrm{~ms}$ \\
\hline TTT (ms) & 160 \\
\hline A3 offset & $3 \mathrm{~dB}$ \\
\hline CoMP Threshold & $6 \mathrm{~dB}$ \\
\hline
\end{tabular}

\begin{tabular}{|c|c|}
\hline UE speed $(\mathrm{km})$ & $3,5,10,20,30$ \\
\hline MeNB to PeNB distance & ISD $>100 \mathrm{~m}$ \\
\hline PeNB to PeNB distance & ISD $>50 \mathrm{~m}$ \\
\hline Handover preparation time & $50 \mathrm{~ms}$ \\
\hline
\end{tabular}

The UE calculates the RSRP every $40 \mathrm{~ms}$, and based on the handover criteria, it generates an MR message that is sent to the serving eNB. We simulated EHoLM and the conventional handover process as mentioned in the previous section using different scenarios. The simulation results are discussed in the following section.

\section{SIMULATION RESULTS}

In order to be able to analyze the potential of the proposed handover procedure over conventional handover procedure, we have simulated both the proposed and the conventional handover process as mentioned in the previous section. We considered different simulation scenarios with varying numbers of PeNBs and users. The initial simulation assumptions are shown in table 1 . We run multiple simulations for each of the scenarios and the simulation results are presented by considering a margin of error for $95 \%$ confidence interval.

Figure 7 shows a comparison between the conventional and EHoLM with respect to the frequency of handover as a function of number of UEs. In this case, we considered one macro cell with 24 pico cells as shown in figure 6(a) and different set of UEs $(25,50,100$ and 200). The speed of the UEs is considered $3 \mathrm{~km} / \mathrm{h}$ and the UEs move at random over the coverage area. The simulation time for all the four sets of UEs is the same. In 7(a), both the conventional and EHoLM handover procedure use the same carrier frequency of $2000 \mathrm{MHz}$ for macro and pico eNBs as suggested in [7] for HetNet scenario 1. In 7(b), we use the same carrier frequency $2000 \mathrm{MHz}$ for macro and pico eNBs in the conventional approach, but carrier frequencies of 2000 and $3500 \mathrm{MHz}$ for EHoLM. In 7(c), both conventional and EHoLM use different carrier frequency $2000 \mathrm{MHz}$ and $3500 \mathrm{MHz}$ for macro and pico eNBs respectively as suggested by 3 GPP in [7] for HetNet scenario 2. All the three cases show that EHoLM reduces the number of handovers significantly.

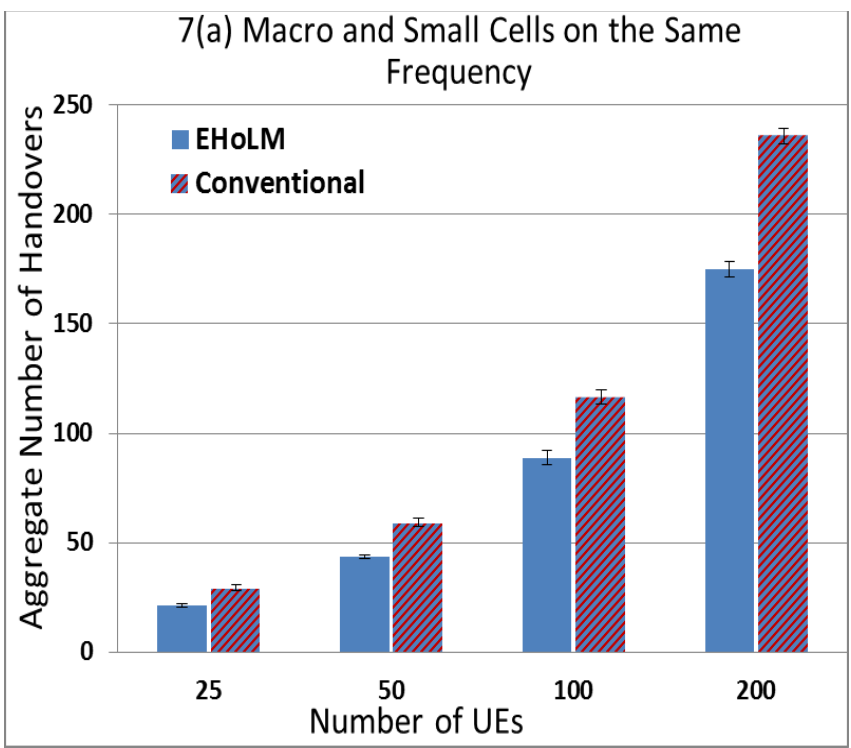


7(b) Macro and Small Cells on Same Frequency in Conventional Approach but Different Frequency in Proposed Approach
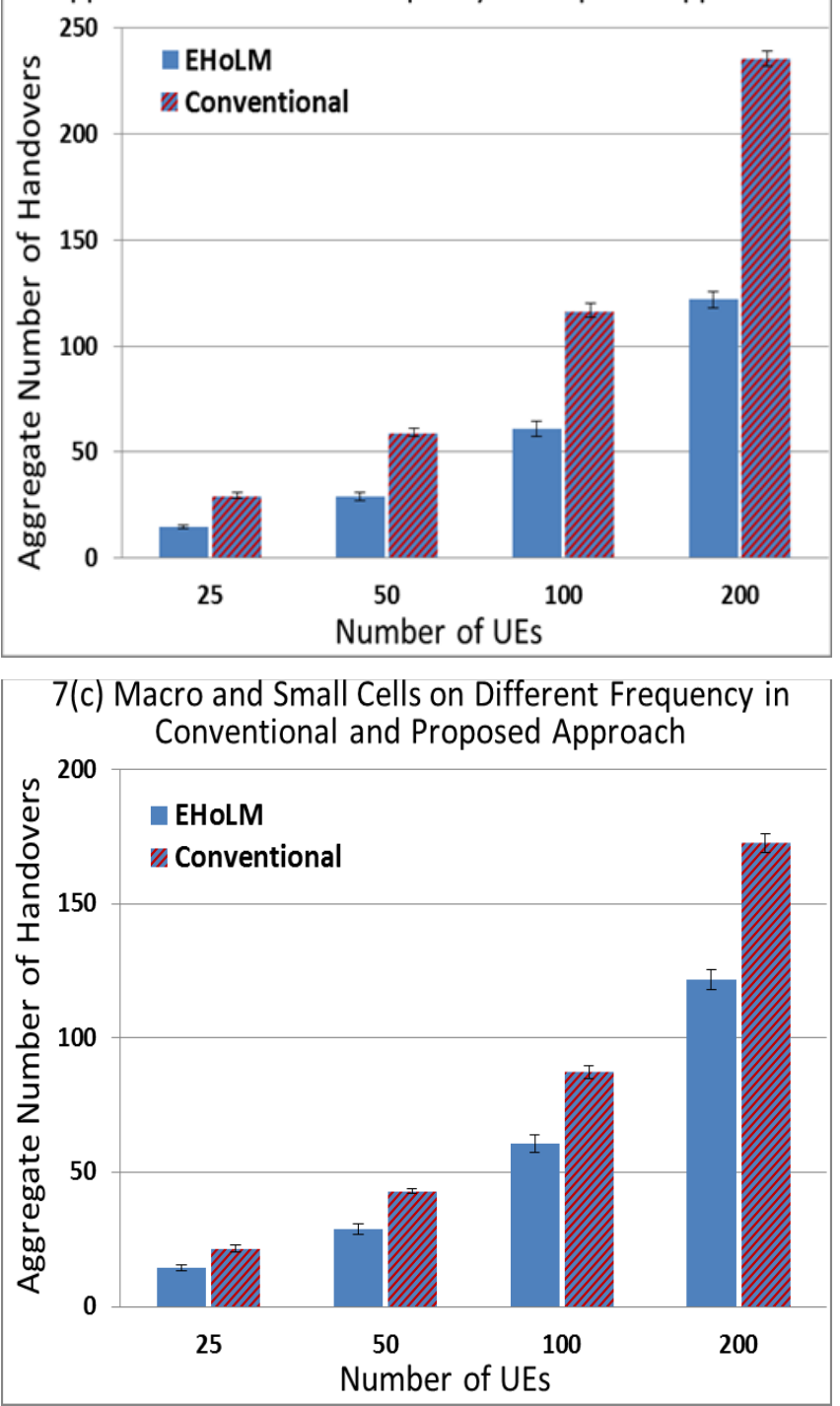

Fig. 7. Number of handovers with respect to number of UEs

Figure 8 shows a comparison between the conventional and EHoLM with respect to the number of handovers as a function of the UE speed. The simulation scenario uses 19 macro cells, 72 Pico cells as shown in figure 6(b) and 200 UEs. The speed of the UEs is considered $3,5,10,20$ and $30 \mathrm{~km} / \mathrm{h}$. The UEs move at random directions over the simulation area from their current position to their destination. In 8(a), both the conventional and EHoLM handover procedures use the same carrier frequency of $2000 \mathrm{MHz}$ for macro and pico eNBs as suggested by $3 \mathrm{GPP}$ for HetNet scenario 1 [7]. In 8(b), both the conventional approach and EHoLM use different carrier frequencies of 2000 and $3500 \mathrm{MHz}$ for macro and pico eNBs respectively as suggested by $3 \mathrm{GPP}$ for HetNet scenario 2 [7]. In 8(c), we use the same carrier frequency of $2000 \mathrm{MHz}$ for macro and pico eNBs in the conventional approach but carrier frequencies of $2000 \mathrm{MHz}$ and $3500 \mathrm{MHz}$ in EHoLM. All the three cases with different UE speed show the EHoLM handover procedure reduces the handover significantly. 8(a) Macro and Small Cell with Same Frequency
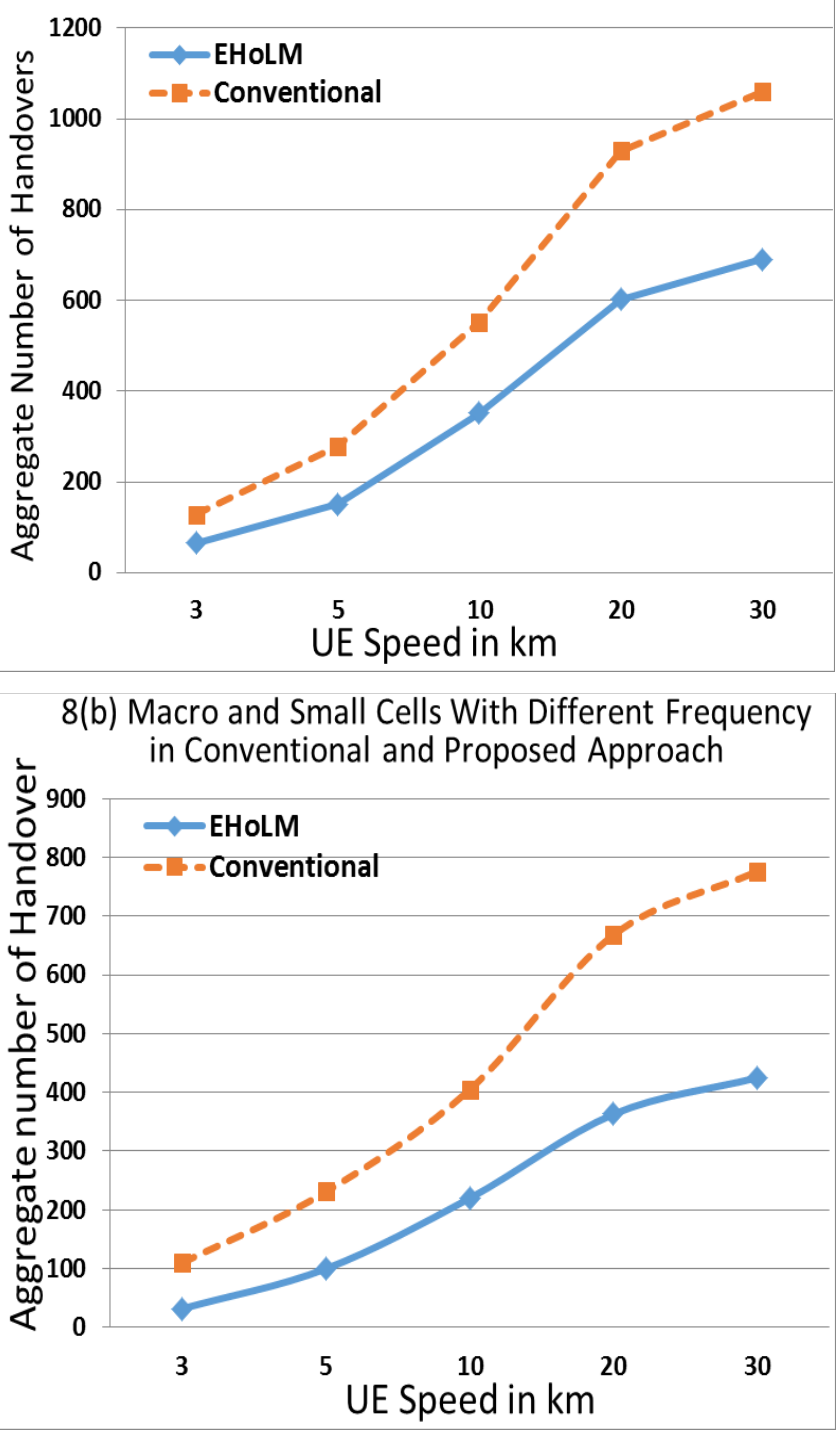

8(c) Macro and Small Cells on Same Frequency in Conventional Approach but Different Frequency in Proposed Approach

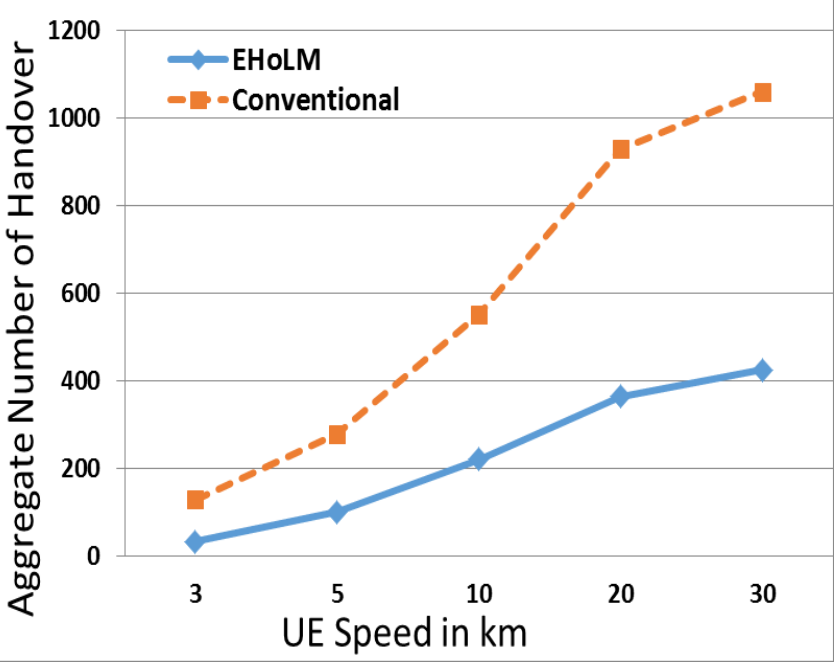

Fig. 8. Number of handovers with respect to UE speed 


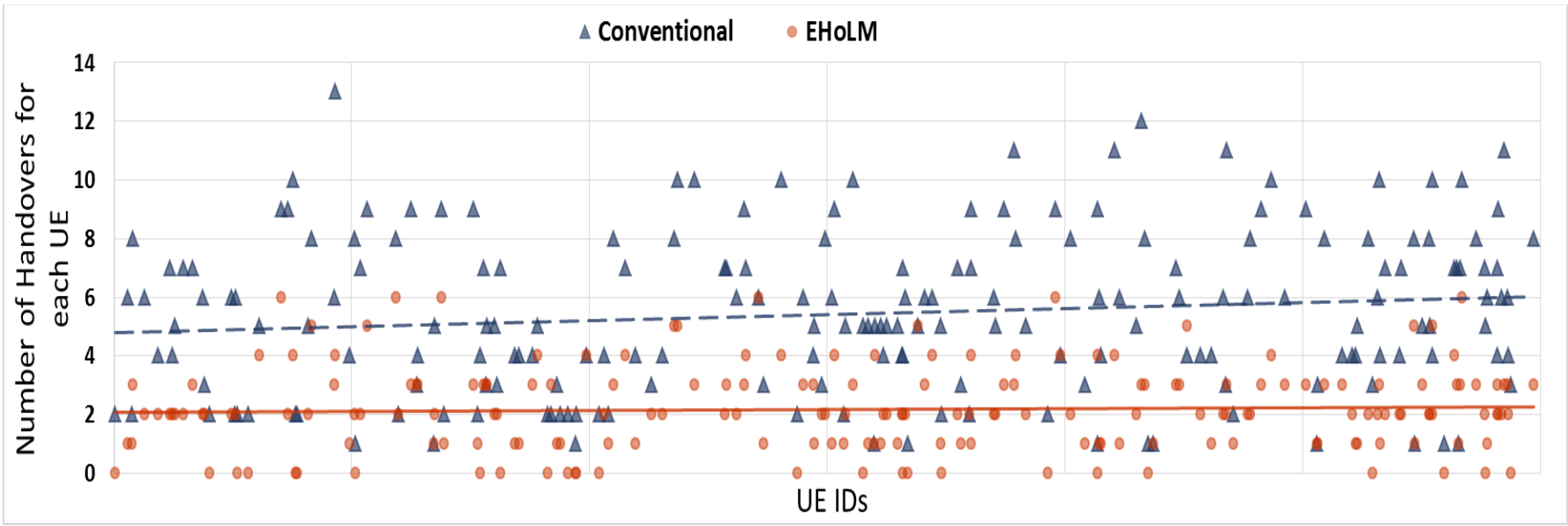

Fig. 9. Number of handovers with respect to each of the UE

Figure 9 shows the number of handovers required for each of the UE in EHoLM and the conventional handover approach. In this case, we also used 19 macro cells, 72 Pico cells and 200 UEs. The blue triangles and the orange circles represent the same UEs in conventional and EHoML approaches respectively. The same UE shifted its position in the graph based on the number of handovers in two different approaches. If we look at the trend lines, it shows that EHoLM reduces about $50 \%$ of handovers than the conventional approach.

According to the simulation results, shown in Figures 7, 8 and 9 we can see that EHoLM has the potential to reduce the number of handovers, which is one of the main performance metrics for evaluating the handover process in heterogeneous cellular networks. The reduction of handover will reduce the signaling overhead and switching load within the cellular network. The signaling overhead is directly impacts to the system performance. Therefore, EHoLM could improve the performance of cellular networks and user experience.

\section{CONCLUSION AND FUTURE WORK}

The main goal of this research is to improve the UE mobility so that network performance could be increase and users get better experience. EHoLM is tested in different heterogeneous scenarios as mentioned in the previous sections of the paper. We have also showed that this approach reduces the number of handover compared to the conventional approach. The reduction of the number of handovers reduces the control overhead within the network. In addition, the reduction of the handover failure improves the users' experience. Therefore, EHoLM has the potential to improve the overall performance of cellular networks. A possibility to expand this work is to study how it affects the handover oscillation in small cells. It could be further expanded to examine the power consumption of the UEs, power consumption of a devices also depends on the message transmission. In the EHoLM handover process, UEs need to transmit less MR than with a conventional approach, having the potential to improve energy efficiency. The energy efficiency is another goal of the next generation cellular networks.

\section{REFERENCES}

[1] Ericsson, "Ericsson Mobility Report," 2016. [Online]. Available: http://www.ericsson.com/res/docs/2015/mobility-report/ericssonmobility-report-nov-2015.pdf. [Accessed 26 March 2016].

[2] M. Peng, Y. Li, Z. Zhao and C. Wang, "System architecture and key technologies for $5 \mathrm{G}$ heterogeneous cloud radio access networks," IEEE Network, vol. 29, no. 2, pp. 6-14, 2015.

[3] M. H. Alsharif and R. Nordin, "Evolution towards fifth generation (5G) wireless networks: Current trends and challenges in the deployment of millimetre wave, massive MIMO, and small cells," Telecommunication Systems, pp. 1-21, 2016.

[4] N. Panwar, S. Shantanu and A. K. Singh, "A survey on 5G: The next generation of mobile communication," Panwar, Nisha, Shantanu Sharma, and Awadhesh Kumar Singh. "A surPhysical Communication, vol. 18, pp. 64-84, 26.

[5] C.-X. Wang, F. Haider, X. Gao, X.-H. You, Y. Yang, D. Yuan, H. Aggoune, H. Haas, S. Fletcher and E. Hepsaydir, "Cellular architecture and key technologies for $5 \mathrm{G}$ wireless communication networks," IEEE Communications Magazine, vol. 52, no. 2, pp. 122 - 130, 2014.

[6] E. Hossain, M. Rasti, H. Tabassum and A. Abdelnasser, "Evolution toward 5G multi-tier cellular wireless networks: An interference management perspective," IEEE Wireless Communications, pp. 118-127, 2014.

[7] 3GPP, "3GPP TR 36.842 V12.0: Study on Small Cell enhancements for E-UTRA and E-UTRAN; Higher layer aspects," December 2013. [Online]. Available: http://www.3gpp.org/DynaReport/36-series.htm. [Accessed July 2016].

[8] V. Jungnickel, K. Manolakis, W. Zirwas, B. Panzner, V. Braun, M. Lossow, M. Sternad, R. Apelfröjd and a. T. Svensson, "The role of small cells, coordinated multipoint, and massive MIMO in 5G.," Communications Magazine, vol. 52, no. 5, pp. 44-51, 2014.

[9] H. Ishii, K. Yoshihisa and T. Hideaki, "A novel architecture for LTE-B: C-plane/U-plane split and phantom cell concept," in Ishii, Hiroyuki, Yoshihisa Kishiyama, and Hideaki Takahashi. "A novel architecture for LTE-B: IEEE Globecom Workshops, 2012.

[10] D. Lopez-Perez, İ. Güvenc and a. X. Chu., "Mobility management challenges in 3GPP heterogeneous networks," Communications Magazine, vol. 50, no. 12, pp. 70-78, 2012.

[11] 3GPP, "3GPP TS 36.300 V14: Evolved Universal Terrestrial Radio Access Network (E-UTRAN)," September 2016. [Online]. Available: http://www.3gpp.org/DynaReport/36-series.htm. [Accessed October 2016].

[12] M. Ding and H. Luo, Multi-point Cooperative Communication Systems: 
Theory and Applications, Shanghai Jiao Tong University Press, Shanghai and Springer-Verlag Berlin Heidelberg, 2013.

[13] S. Sun, Q. Gao, Y. Peng, Y. Wang and L. Song, "Interference Management Through CoMP in 3GPP LTE-Advanced Networks," IEEE Wireless Communications, vol. 20, no. 1, pp. 59-66, 2013.

[14] J. Lee, Y. Kim, H. Lee, B. L. Ng, D. Mazzarese, J. Liu, W. Xiao and Y. Zhou, "Coordinated Multipoint Transmission and Reception in LTEAdvanced Systems," IEEE Communications Magazine, vol. 50, no. 11, pp. 44-50, 2012.

[15] S. C. Jha, S. Kathiravetpillai, V. Rath and A. T. Koc., "Dual connectivity in lte small cell networks," in IEEE Globecom Workshops (GC Wkshps), Austin, Texas, USA, 2014.

[16] 3GPP, "3GPP TR 36.819 version 11.2.0: Coordinated multi-point operation for LTE physical layer aspects," 09 2013. [Online]. Available: http://www.3gpp.org/DynaReport/36-series.htm. [Accessed September 2016].

[17] B. U. Kazi, M. Etemad, G. Wainer and G. Boudreau, "Using Elected Coordination Stations for CSI Feedback on CoMP Downlink Transmissions," in International Symposium on Performance Evaluation of Computer and Telecommunication Systems, Montreal, Canada, 2016.

[18] B. Özbek and D. L. Ruyet, "Feedback Strategies for Multicell Systems," in Feedback Strategies for Wireless Communication, New York, Springer, 2014, pp. 249-293.

[19] Y. Gao, Y. Li, H. Yu and a. S. Gao., "Performance analysis of dynamic CoMP cell selection in LTE-advanced heterogeneous networks scenario," in International Conference on Uncertainty Reasoning and Knowledge Engineering (URKE), Bali, Indonesia, 2011.

[20] S. Geirhofer and a. P. Gaal, "Coordinated multi point transmission in 3GPP LTE heterogeneous networks," in In Globecom Workshops (GC Wkshps), Anaheim, CA, 2012 .

[21] B. U. Kazi, M. Etemad, G. Wainer and G. Boudreau, "Signaling Overhead and Feedback Delay Reduction in Heterogeneous Multicell Cooperative Networks," in International Symposium on Performance Evaluation of Computer and Telecommunication Systems, Montreal, Canada, 2016.

[22] 3GPP, "3GPP TR 36.932 V13: Scenarios and requirements for small cell enhancements for E-UTRA and E-UTRAN," 2016-01. [Online]. Available: http://www.3gpp.org/DynaReport/36-series.htm. [Accessed August 2016].

[23] 3GPP, "3GPP TS 23.0009 V13: Handover procedures," 2015-12. [Online]. Available: http://www.3gpp.org/DynaReport/23-series.htm. [Accessed July 2016].

[24] G. A. Wainer, Discrete Event Modeling and Simulation A Practitioner's approach, Boca Raton, FL: CRC Press, Taylor \& Francis Group, 2009.

[25] G. A. Wainer, M. Tavanpour and E. Broutin, "Application of the DEVS and Cell-DEVS formalisms for modeling networking applications," in Proceedings of the Winter Simulation Conference (WSC), Washington, DC, 2013

[26] B. Zeigler, H. Praehofer and T. Kim, Theory of modeling and simulation, San Diego, CA: Academic Press, 2000.
[27] A. Inostrosa-Psijas, G. Wainer, V. Gil-Costa and M. Marin, "DEVS Modeling of Large Scale Web Search Engines," in Proceedings of the 2014 Winter Simulation Conference, Savannah, GA, 2014.

[28] 3GPP, "3GPP TR 36.839 V11.1: Evolved Universal Terrestrial Radio Access (E-UTRA); Mobility enhancements in heterogeneous networks," 12 2012. [Online]. Available: http://www.3gpp.org/DynaReport/36series.htm. [Accessed July 2016].

[29] 3GPP, "3GPP TS 36.133 V14: Evolved Universal Terrestrial Radio Access (E-UTRA); Base Station (BS) and repeater ElectroMagnetic Compatibility (EMC)," 2016-06. [Online]. Available: http://www.3gpp.org/DynaReport/36-series.htm. [Accessed August 2016].

[30] K. Vasudeva, M. Simsek, D. López-Pérez and I. Guvenc, "Analysis of Handover Failures in Heterogeneous Networks with Fading," Vasudeva, Karthik, Meryem Simsek, David López-Pérez, and Ismail Guvenc. "Analysi arXiv preprint arXiv:1507.01586, 2015.

[31] Q. Kuang, B. Jakob, B. Zarah, D. Heinz and S. Joachim, "A measurement-based study of handover improvement through range expansion and interference coordination," Wireless Communications and Mobile Computing, vol. 15, no. 14, pp. 1784-1798, 2015.

[32] 3GPP, "3GPP TR 36.819 version 11.2.0: Coordinated multi-point operation for LTE physical layer aspects," 09 2013. [Online]. Available: http://www.3gpp.org/DynaReport/36-series.htm. [Accessed September 2016].

[33] X. Zhang and X. Zhou, LTE-Advanced Air Interface Technology, CRC Press, 2012.

[34] 3GPP, "3GPP TR 36.842 V12.0: Study on Small Cell enhancements for E-UTRA and E-UTRAN; Higher layer aspects," 2013-12. [Online]. Available: http://www.3gpp.org/DynaReport/36-series.htm. [Accessed July 2016].

[35] 3GPP, "3GPP TS 36.814 V9.0: Evolved Universal Terrestrial Radio Access (E-UTRA); Further advancements for E-UTRA physical layer aspects," 2010-03. [Online]. Available: http://www.3gpp.org/DynaReport/36-series.htm. [Accessed July 2016].

[36] G. Cili, H. Yanikomeroglu and F. R. Yu, "Cell Switch Off Technique Combined with Coordinated Multi-Point (CoMP) Transmission for Energy Efficiency in Beyond-LTE Cellular Networks," in Communications (ICC), 2012 IEEE International Conference on, Ottawa, ON, 2012.

[37] 3GPP, "3GPP TR 36.942 V13.0: Evolved Universal Terrestrial Radio Access (E-UTRA); Radio Frequency (RF) system scenarios," 012016. [Online]. Available: http://www.3gpp.org/DynaReport/36-series.htm. [Accessed April 2016]. 